Dept. of Immunity \& Bacteriology,

Animal Health Research Institute, Mansora Branch.

\title{
BACTERIOLOGICAL AND SEROLOGICAL STUDIES ON PASTEURELLA MULTOCIDA INFECTION IN RABBITS
}

By

\section{A.M.E. ASWAY; A.M. AHMED and H.M.H. SHALBY* \\ * Dept. of Bacteriology, Animal Health Research Institute, Dokki.}

(Received at 24/5/2008)

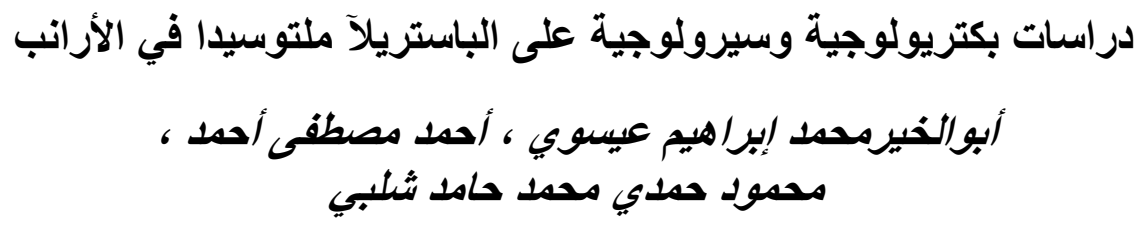

الباستريلا ملتو سيد ا ميكروب مخاطي يستعمر الجزء العلوي من الجهاز التنفسي للأر انب.

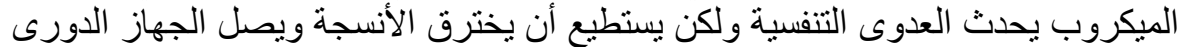

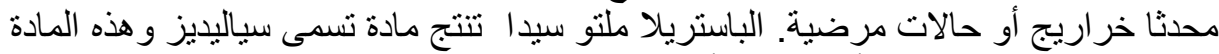

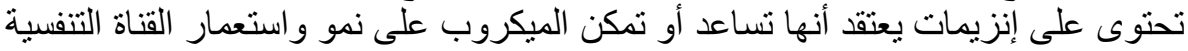

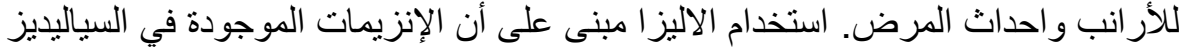

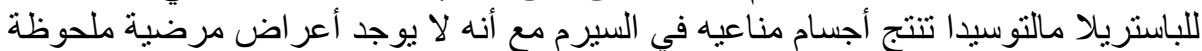

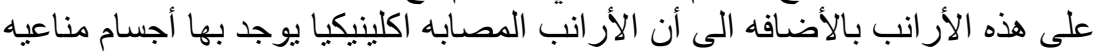

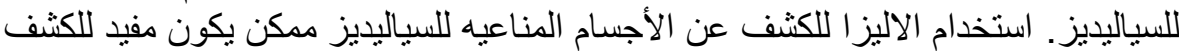

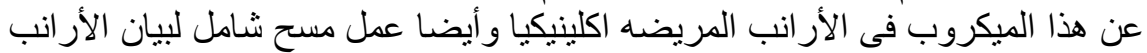

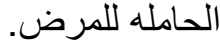

\section{SUMMARY}

Pastruella multocida is a mucosal pathogen that colonizes the upper respiratory tract of rabbits. Respiratory infections can result, but also the bacteria can invade the circulatory system producing abscesses or septicemia. Pasteurella mulocida (P.multocida) produce extracelluler sialidase that encode enzymes which is believed to augment colonization of the respiratory tract and the production of lesions in the active infection. The recombinant antigen enzyme-linked immunosorbent assay (ELISA) based on the sialidas enzyme of P.multocida and rabbits that were experimentally colonized with P.multocida produce detectable immunoglobulin $(\operatorname{IgM})$ and $(\operatorname{IgG})$ in serum, although they demonstrated no clinical signs of pasteurellosis. In addition clinically ill pet rabbits 
infected with P.multocida possessed IgM and/orIgG antibody against sialides. The sialides ELISA may be useful for the diagnosis of P.mulocida infection in sick rabbits as well as for screening for carriers rabbits.

Key words: Rabbits, Pasteurella multocida, ELISA.

\section{INTRODUCTION}

Pasteurella multocida can be a virulent pathogen of rabbits producing fatal septicemia,pneumonia,chronic rhinitis and otitis media as well as multiple abscesses, however some rabbits are persistently colonized and exhibit no apparent signs of disease (Lu, et al., 1978). Many rabbits become colonized with

Pasteurella soon after birth and after weaning more than $75 \%$ of rabbits that nurse from infected dams become culture positive (Holmes, et al., 1984). The prevalence of P.multocida in clinically healthy rabbits ranges from $20-90 \%$, Depending on the methods used for detection, as well as the age health status of rabbits (Glass and Beasley, 1989 and Lu, et al., 1978). Laboratory rabbits colonized with P.multocida often develop clinical disease after being shipped to a research facility, but persistently colonized asymptomatic rabbits have been shown to produce aberrant results if they are used in research (Richard, et al., 1997). The effect of pasteurellosis on biomedical research is so profound that continuous screening of research rabbits colonies is recommended (Ward, G.W. 1973). Culture of nasal swab specimens has been shown to be unreliable for screening, since up to $30 \%$ of infected rabbits may not be detected by this method (Holmes, et al., 1986 and Holmes, et al., 1987). In addition, serological screening has not been effective in identifying all persistently colonized rabbits because most of the serological tests use uncharacterized antigen mixtures that may not detect the multitude of serotypes that colonize rabbits (Delong, et al., 1992; Kawamoto, et al., 1994; Klassen, et al., 1985; Lukas, et al., 1987. Manning, P.J. 1984 and Zaoutis, et al., 1991) Vaccination is not commercially available because of lack of efficacy, and furthermore, antibiotics may be effective for resolving the symptoms in sick rabbits but usually do not clear the bacteria from colonized rabbits (Gaertner, D.J. 1991; Mahler, et al., 1995 and Welch, et al., 1987). P.multocida isolates vary in their abilities to produce disease in rabbits, some are associated primarily with upper respiratory disease, while others cause septicemia, abscesses and pneumonia (DiGiacomo, et al., 1991). 
However, in order to initate infection, the bacteria must colonize the respiratory mucosa, and organisms that inhabit mucosal surfaces frequently produce sialidases (Corfield, T. 1992 and Vimer and Lichtensteiger, 2002). Sialidase is the only extra-cellular glycolytic enzyme probably plays a major role in the ability of P.multocida to colonize animals (Drazeniek, et. al., 1972; Lee, et al., 1988 and Scharmann, et al., 1970), suggesting that this antigen may be useful for the serological diagnosis of pasteurellosis. In this study we report on the use of enzyme-linked immunosorbent assay (ELISA) to detect P.multocida infection in healthy and clinically ill rabbit.

\section{MATERIALS and METHODS}

Bacterial field isolate: 40swabs of lesions or nasal exudates with serum samples were acquired from rabbits from different localities at sharkia governorate with clinical signs suggestive of pasteurellosis. These swabs were streaked onto blood and chocolate agar plates for P.multocida (Knight, et al., 1983 and Avirl, et al., 1990)

Reference strain: P.multocida reference strain were obtained from veterinary serum and vaccine research institute, abbasia, Cairo.

Experimental animal: 13 male New Zealand rabbits 4-6week of age were used for production of antibodies and challenge.

Production of antibodies: Antibodies against P.multocida was produced by intradermal injection of $200 \mathrm{ug}$ of purified antigen in 500ul of complete freund adjuvant in 20 different sites on one New Zealand white male rabbit. The rabbit was vaccinated twice subcutaneously with 100ug of antigen in Freunds incomplete adjuvant at 3 weeks intervals. Preimmune blood samples were collected before the $1^{\text {st }}$ immunization and serum was collected 14 day after each vaccination (Brogden, et al., 1983).

Rabbit challenge: 12 male New Zealand white rabbits (4-6weeks of age) were obtained from Pasteurella free vendor. The rabbits were housed in stainless steel cages. $150 \mathrm{gm}$ of commercial pelleted feed was provided daily and water was available ad libitum. Serum samples were collected from 12 rabbits, 2 weeks after they were housed, in order to establish a negative baseline for ELISA. The rabbits were divided into 3 groups (each of 4 rabbits), the first group were kept as control without any treatment, the second group were administered intranasally $10 \mathrm{ul}$ of phosphate buffer saline containing approximately $10 \mathrm{CFU}$ of reference strain, these rabbits become systemically ill within 30 hours post 
exposure and were administered $10 \mathrm{mg}$ of enrofloxacin $/ \mathrm{kg}$ body weight subcutaneously twice daily for 5 days. Serum was collected from the rabbits prior to challenge and weekly thereafter for the next 5 weeks. and the third group with the field isolates, serum was collected from the rabbits prior to challenge and weekly thereafter for the next 11 weeks. On week 12, the rabbits were sacrificed and then sample swabs were taken from the deep nasal turbinates, pharynx, trachea, ear bullae and cervical lymph nodes for culture. A full necropsy was performed on all of the rabbits in order to detect gross indications of infections.

ELISA: a whole cell-lysate ELISA was performed as described by (kawamoto, et al., 1990 \& 1994 and Bonga, et al., 1997) in order to confirm the infection status of rabbits.

\section{RESULTS and DISCUSSION}

The term pasteurellosis can be used to refer several condition caused by P.multocida that harbored in the nasal cavity of rabbits leading to rhinitis or asymptomatic chronic infection. The importance of pasteurellosis in rabbits is underscored by the fact that its clinical syndromes are refractory to antibiotic administration and there no reported vaccination strategy that can prevent all the manifestation of the disease (Manning, et al., 1994).

Colonization and disease is influenced by factors related to both host and pathogen. Three strains of P.multocida have been isolated from rabbits. They are classified by capsular type and serotype into A:12, A:3, $2: \mathrm{D}$ and $\mathrm{B}$ in which $\mathrm{A}: 12$ is the most common in rabbits but the more sever disease has been associated with A:3.

The reference serotype and field isolates of P.multocida tested produce sialidase activity in the filter paper spot test (Mizan. et. al., 2000).

All isolates including the reference isolate suggesting that this antigen is widely distributed among the serotypes of P.multocida when they were used in whole-cell ELISA, suggesting that all serotypes tested produce an antigenically conserved the antigen (sialidase). These results suggest that sialdise may be useful antigen for the serological screening of rabbits infected with P.multocida.

Sialidase gene (antigen) has been shown to be difficult to purify from P.multocida, because it is membrane associated (Mizan, et al., 2000). When this antigen protein was used to vaccinate a rabbits, high titer antibodies serum was acquired. The serum antibodies was used as 
positive control serum in the ELISA. All rabbits used in this experiment were nasal swab culture and ELISA negative for P.multocida and demonstrated no symptoms of disease prior to testing by ELISA or challenge. Sera from these rabbits were tested for cross-reactive antibodies against this antigen by ELISA. Science cross-reactive antibodies were most apparent at the lower dilutions, we chose dilution of $1 / 16(\operatorname{IgG})$ and $1 / 18(\operatorname{IgM})$ as the minimal dilutions for the detection of antibody titers.

Serum from vaccinated rabbits, which was exsanguinated 2 weeks after the third immunization served as the positive control for the IgG ELISA, serum removed from these rabbits 2 week after the first immunization served as the positive control serum for IgM ELISA and the sera from the negative rabbits were also used as the negative control for the antibodies (IgM) ELISA.

The immune system of colonized rabbits may have become exposed to P.multocida surface protein during colonization and bacterial cells may also have been phagocytosed by antigen processing cells present in the mucosal tissue. In order to determine whether antibody could be detected in colonized rabbits we challenged rabbits intranasally with several P.multocida isolates. 3rabbits $\left(2^{\text {nd }}\right.$ group) that are administered highly virulent (reference) isolate exhibited symptoms of septicemia on the day after challenge and three rabbits ( $3^{\text {rd }}$ group) that are administered the field isolates (cocktail) show symptoms of snuffles. The rabbits of $2^{\text {nd }}$ groups were treated with antibiotic that resolved the infection, and all of the rabbits recovered. Serum from the rabbits did not display antibodies, and P.multocida was not detected post-treatment by ELISA or culture. However two of the four rabbits from this group that were tested by whole cell-lysate ELISA produced positive titers 2-3 week post challenge, confirming their P.infection status. The rapid onset of disease, coupled with therapeutic treatment, probably resulted inadequate exposure to P.multocida surface sialidase and the antibiotics have been only partially successful in controlling infection, since they do not completely eliminate the bacterium (Gaertner, 1991 and Jaslow, et al., 1981).

In contrast, the rabbits that were administrated a cocktail of the field isolates ( $3^{\text {rd }}$ group) produce $\operatorname{IgM}$ antibodies and one rabbit demonstrated IgG antibodies 6 weeks after inoculation. Since we used anti-whole-molecule for detection, the IgG titer was actually nonisotype specific IgG. The IgG levels continued to rise in these rabbits, but one rabbit exhibited a positive titer only after 11 weeks postexposture. IgM 
titers were transient and undetectable in the samples collected at 9 weeks postexposture .P.multocida was detected in rabbits of these group 14 week post inoculation by ELISA and nasal swabs collected.

At necropsy P.multocida was also isolated by culture from tracheal swab specimen of rabbits that demonstrated the highest $\operatorname{IgG}$ levels post inoculation.

Neither rabbit exhibited any clinical signs of P.multocida infection, nor were lesion detected by gross pathology, suggesting that the rabbit that colonized but not adversely affected by the bacteria. While serum samples from these rabbits produced increasing values by Kawamoto ELISA over the course of the experiment, only one serum sample from one rabbit had a positive titer.

These results suggest that this antigen (sialidase) is sufficiently expressed by P.multocida during surface colonization of the respiratory mucosal system to stimulate serum antibody production. Sera and swabs collected from control group were negative for ELISA and cultures. These results suggest that the ELISA may be useful for identifying healthy carrier rabbits. Because of its virulence, early diagnosis of P.multocida infection in pet rabbits is critical. Exposure to virulent invasive strains may result in rapid penetration of the respiratory mucosa, leading to per acute or acute disease (Al-Hadawi, et al., 1999; Al-Haddawi, et al., 2001; Lee, et al., 1994 and Rabier, et al., 1997). In the challenge with reference strain described in this report, we observed rapid infection, in which the rabbits were clinically ill within $30 \mathrm{~h}$. after intranasal exposure to a low bacterial dose. Similar results have been reported by others using other virulent P.multocida isolates in rabbits (Al-Haddawi, et al., 2000; Al-Haddawi, et al., 2001 and Al-Lebban, et al., 1988). These infection might be detectable by PCR or culture because of the high replication rate of bacteria in the tissue (Lee, et al., 1988). Although culture is considered the gold standard for detection ,techniques for the isolation and identification of P.multocida are time consuming and often fail because some transport media, including commonly used for transport swabs, do not maintain P.multocida viability for more than 1day at room temperature (Kawamoto, et al., 1997). In some clinical cases, the organism can not be cultured from obviously diseased organs because the animals may have received antibiotics prior to sample submission. In sick animals that exhibit per acute disease, an ELISA may not be useful for the detection of antibodies post infection if the animals have been treated with antibiotics. 
It is also important to determine healthy research rabbits are P.multocida free. The bacteria can be present at low numbers deep in the nasal turbinates of carrier rabbits, rendering their detection by culture impossible without killing the animal (Holmes, et al., 1987 and Ward, G.M.1973). Several research groups have investigated ELISA as a method for the detection of colonization of healthy rabbits with P.multocida (Holmes, et al.1986; Hwang, et al., 1986; Kawamoto, et al., 1994; Klassen, et al., 1985; Lukas, et al., 1987; Peterson, et al., 1997 and Zaoutis, et al., 1991). However, rabbits exposed to low-virulence strains may become persistently colonized (DeLong, et al., 1992), but the bacteria may be primarily associated with the mucus layer or may be adhere to the surface of mucosal epithelial cells (Al-Hddawi, et al., 2000; Glorioso, et al., 1982). The ELISA can detect this colonization state in chronically colonized animals. (West, et al., 2002) demonstrated that serology with extra cellular proteins or cell lysates could detect colonization 6-12 months before culture detection, analogous to the screening of rabbits for P.multocida infection.

Stress or shipping can reduce rabbit mucosal defenses and allows the bacteria to invade the mucosal surface, with subsequent host exposure to other bacterial antigens. Low virulence strains would be more likely to produce chronic infections, which would result in high anti-P.multocida antibody titers in serum that could be detected by serological methods. Most serological tests use boiled whole P.multocida cells, heat-stable cell lysates, or purified lipopolysaccharides (LPS) as the antigen, which can result in both high background levels and significant numbers of false-positive or falsenegative results (Cary, et al., 1984; Hwang, et al., 1986; Manning, P.G. 1984 and Manning, et al., 1986). Infected rabbits may make high levels of LPS-specific antibody (Cary, et al., 1984; Manning, P.G. 1984 and Manning, et al., 1986) But the diversity of P.multocida serotype-specific LPS ensures that some strains may not be reactive by all assays (Brogden and Packer, 1979; Chengappa, et al., 1982 and Rimler and Brogden, 1986)

A better target for P.multocida detection would be a homogeneous antigen that could be easily purified and that is present in all isolates. In order to obtain a more homogeneous antigen for serological testing, purified recombinant sialidase genes are used. It was shown that sialidase is associated with P.multocida outer membrane and dose not exhibit a high degree of homology to other sialidases that have been characterized (Mizan, et al., 2000). 
In the present study, we demonstrated that the antigen is ubiquitous in P.multocida isolates that cause disease in domestic animals. Many organisms that colonize the respiratory system produce sialidase, and this enzyme functions in the removal of sialic acid from mucus, which allows sialidase- producing bacteria such as P.multocida to access sialic acid as an energy source (Corfield, 1992; Mizan, et al., 2000 and Vimer, and Lichtensteiger, 2002). We have shown that expression of sialidase during colonization or chronic infection of deep host tissue elicits humoral response, which enables detection of persistent colonization by serology.

\section{REFERENCES}

Al-Haddawi, M.H.; Jasni, S.; Zamri-Saad, M.; Mutalib, A.R. and Sheikh-Omer, A.R. (1999): Ultra structural pathology of the upper respiratory tract of rabbits experimentally infected with Pasteurella multocida A:3.Res.Vet.Sci.67:163-170.

Al-Haddawi, M.H.; Jasni, S.; Zamri Saad, M.; Mutalib, A.R.; Zulkiffi, I.;

Son, R. and Shikh-Omar, A.R. (2000): In vitro study of

Pasteurella multocida adhesion to trachea, lung, and aorta of rabbits. Vet. J. 159: 274-281.

AL-Haddawi, M.H.; Jasni, S.; Israf, D.A.; Zamri Saad, M.; Mutalib, A.R. and Shi-kh-Omar, A.R. (2001): Ultra structural pathology of nasal and tracheal mucosa of rabbits experimentally infected with Pasteurella multocida serotype D: 1. Res. Vet. sci. 70: 191-197.

Al-Lebban, Z.S.; Corbeil, L.B. and Coles, E.H. (1988): Rabbitpasteurellosis: induc-ed disease and vaccination. Am. J. Vet. Res. 49: 312-316.

Avirl, J.; Donno, P. and Pouedras, P. (1990): Selective medium for Pasteurella multocida and its use to detect oropharyngeal carriers in pig breeders. J. Clin. Microbiol. 28: 1438-1440.

Boga, B.O.; Kedrak, A. and Truszczynski, M. (1997): Application of the ELISA for determination of anti-P.multoida IgG in the sera of rabbits vaccinated against pasteurellosis under field conditions. Bull. Vet. Inst. Pulawy 41: 17-23.

Brogden, K.A. and Rhoades, K.R. (1983): Prevalence of serologic types of P.mutocida from 57 species of birds and mammals in the United States. J. Wild. Dis., 19: 315-320. 
Brogden, K.A. and Packer, R.A. (1979): Comparison of Pasteurella multocida serotyping systems. Am. J. Vet. Res. 40: 1332-1335.

Cary, C.J.; Per, J.K.; Chrisp, C.E. and Keren, D.F. (1984): Serological analysis of live serotypes of Pasteurella multocida of rabbit origin by use of an enzyme-linked immunosorbent assay with lipopolysaccharide as an antigen. J. Clin. Microbiol. 20: 191-194.

Chengappa, M.M.; Myers, R.C. and Carter, G.R. (1982): Capsular and somatic types of Pasteurella multocida from rabbits. Can. J. Comp Med. 46: 437-439.

Corfield, T. (1992): Bacterial sialidasis-roles in pathogenicity and nutrition glycobiology 2: 509-521.

DeLong, D.; Manning, P.J.; Gunther, and Swanson, D.L. (1992): Colonization of rabbits by Pasteurella multocida serum IgG responses following intranasal challenge with serologically distinct isolation. Lab. Anim.Sci. 42: 13-18.

DiGiacomo, R.F.; Xu, Y.M.; Allen, V.; Hinton, M.H. and Pearson, G.R. (1991): Naturally acquired Pasteurella multocida infection in rabbits: clinicopathological aspects. Can.J. Vet. Res. 55: 234-238.

Drzeniek, R.; Scharmann, W. and Balke, E. (1972): Neuraminidase and $\mathrm{N}$-acetylneuraminate pyruvate-lyase of Pasteurella multocida. J. Gen. Microbiol. 72: 357-368.

Gaertner, D.J. (1991): Comparison of penicillin and gentamicin for treatment of pasteurellosis in rabbits. Lab. Anim. Sci. 41: 78-80.

Glass, L.S. and Beasley, J.N. (1989): Infection with and antibody response to Pasteurella multocida and Bordetella bronchieseptica in immature rabbits. Lab. Anim. Sci. 37: 406-410.

Glorioso, J.C.; Jones, G.W.; Rush, H.G.; Pentler, L.J.; Darif, C.A. and Coward, J.E. (1982): Adhesion of type A Pasteurella multocida to rabbit pharyngeal cell and its possible role in respiratory tract infections. Infect. Immun. 35: 1103-1109.

Holmes, H.T.; Patton, N.M. and Cheebe, P.R. (1984): The occurrence of Pasteur-errla multocida in newborn and weaning rabbits. J. Appl. Rabbit Res. 7:17-20.

Holmes, H.T.; Matsumoto, M.; Patton, N.M. and Zehfus, B.R. (1986): Serological methods for detection of Pasteurella multocida 
infections in nasal culture negative rabbits. Lab. Anim.Sci.36: 640-645.

Holmes, H.T.; Matsumoto, M.; Patton, N.M. and Harris, D.J. (1987): A method for culturing the nasopharyngeal area of rabbits. Lab. Anim. 21: 353-355.

Hwang, E.J.; Holmes, H.T.; Zehfus, B.P.; Patton, N.M. and Matsumoto, $M$. (1986): Characterization of antigen purified from from type 3 strains of Pasteurella multocida and its use for an enzymelinked immunosorbent assay. Lab. Anim. Sci. 36: 633-639.

Jaslow, B.W.; Ringler, D.H.; Rush, H.G. and Glorioso, J. (1981): Paseurella associated rhinitis of rabbits: efficacy of penicillin therapy. Lab. Anim. Sci. 131: 382-385.

Kawamoto, E.; Sawada, T.; Suzuki, K. and Maruyama, T. (1990): Serotypes of Pasteurella multocida isolates from rabbits and their environment in Japan.Jpn.J.Vet.Sci. 52: 1277-1269.

Kawamoto, E.; Sawada, T.; Sato, T.; Suzuki, K. and Maruyama, T. (1994): Compa-rison of indirect haemagglutination test, geldiffusion precipitin test, and enzyme-linked immunosorbent assay for detection of serum antibodies to Pasteurella multocida in naturally and experimentally infected rabbits. Lab. Anim. 28: 19-25.

Kawamoto, E.; Sawada, T. and Maruyama, T. (1997): Evaluation of transport media for Pasteurella multocida isolates from rabbit nasal specimens. J. Clin. Micobiol. 35: 1948-1951.

Klassen, J.M.; Bernard, B.L. and DiGiacomo, R.F. (1985): Enzymelinked imm-unosorbent assay for immunoglobulin $\mathrm{G}$ antibody to Pasteurella multocida in rabbits. J. Clin. Microbiol. 21: 617-621.

Knight, D.P.; Paine, J.E. and Speller, D.C.E. (1983): A selective medium for Pasteurella multocida and its use with animal and human specimens. J. Clin. Pathol. 36: 591-594.

Lee, M.D.; Brown, J.; Wooley, R.F. and Glisson, R. (1988): The relationship of pathogenicity to the growth of 3,4 Pasteurella multocida isolates in normal turkey plasma .Avian Dis. 32: 509-512.

Lee, M.D.; Wooley, R.F. and Glisson, J.R. (1994): Invasion of turkey epithelial cell monolayer by Pasteurella multocida Avian Dis. 38: $72-77$. 
Lu, Y.; Ringler, D.H. and Parkes, J.S. (1978): Characterization of Pasteurella multocida isolates from the nares of healthy rabbits and rabbits with pneumonia. Lab. Anim. Sci. 28: 691-697.

Lukas, V.S.; Ringler, D.H.; Chrisp, C.E. and Rush, H.G. (1987): An enzyme-linked immunosorbent assay to detect serum IgG to Pasteurella multocida in naturally and experimentally infected rabbits. Lab. Anim. Sci. 37: 60-64.

Mahler, M.; Stunkel, S.; Ziegowski, C. and Kunstyr, I. (1995): Inefficacy of enrofloxacin in the elimination of Pasteurella multocida in rabbits. Lab. Anim. 29: 192-199.

Manning, P.J. (1984): Naturally occurring pasteurellosis in laboratory rabbits: chemical and serological studies of whole cell and lipopolysaccharides of Pasteurella multocida. Infect. Immun. 44: 502-507.

Manning, P.J.; Patrick; Ringler, H.; Daniel, and NewComer, E.C. (1994): Pasteurellosis; the biology of laboratory rabbits. $2^{\text {nd }}$ Ed. 131-140.

Manning, P.G.; Naasz, M.A.; DeLong, D. and Leary, (1986): Pasteurellosis in laboratory rabbits: characterization of lipopolysaccharides of Pasteurella multocida by polyacrylamide gel electrophoresis, immunoblot techniques and enzyme-linked immunosorbent assay. Infect. Immun. 53: 460-463.

Mizan, S.A.; Henk, D.; Stallings, A.; Meier, M. and Lee, M.D. (2000): Cloning and characterization of sialidases with 2-6' and 2-3' sialyl lactose specificity from Pasteurella multocida. J. Baceriol. 182: 6874-6883.

Peterson, R.R.; Deeb, B.J. and DiGiacomo, R.F. (1997): Detection of antibodies to Psteurella multocida by capture enzyme immunoassay using a monoclonal antibody against P37 antigen. J. Clin. Microbiol. 35: 208-212.

Rabier, M.J.; Tyler, N.K.; Walker, N.J.; Hasen, L.M.; Hirsh, D.C. and Tablin, F. (1997): Pasteurella multocida enters polarized epithelial cells by interacting with host F-actin.Vet.Microbiol. 54: 343-355.

Richard, M.; Reske, M.M.; Delaney, K.; Fletch, A.; Wilcox, L.H. and Kinlough-Rathbone, R.L. (1997): Respiratory infection in lipid-feed rabbits enhances sudanophilia and the expression of VCAM-1 Am. J. Pathol. 151: 1009-1017. 
Rimler, R.B. and Brogden, K.A. (1986): Pasteurella multocida isolated from rabbits and swine; serological types and toxin production. Am. J. Vet. Res. 47: 630-737.

Scharmann, W.; Drzenick, R. and Biobel, H. (1970): Neuraminidase of Pasteurella multocida. Infect. Immun. 1: 319-320.

Vimer, E. and Lichtensteiger, C. (2002): To sialyate, or not to sialyte: that is the question Trends Microbiol. 10: 254-257.

Ward, G.W. (1973): Development of a Pasteurella-free rabbits colony. Lab. Ani-m. Sci. 23: 671-674.

Welch, W.D.; Lu, Y.S. and Bawdon, R.E. (1987): Pharmacokinetics of penicillin-G in serum and nasal washings of Pasteurella multocida free and infected rabbits. Lab. Anim. Sci. 37: 65-68.

West, S.E.; Zeng, L.; Lee, B.L.; Kosorok, M.R.; Laxova, A.; Rock, M.J.; Splaingard, M.J. and Farrell, P.M. (2002): Respitatory infection with Pseudomonas aeruginosa in children with cystic fibrosis: early detection by serology and assessment of risk factors. JAMA 287: 2958-2967.

Zaoutis, T.E.; Reinhard, G.R.; Cioffe, C.J.; Moore, P.B. and Stark, D.M. (1991): Screening rabbit colonies for antibodies to Pasteurella multocida by an ELISA. Lab. Anim. Sci. 41: 419-422. 